\title{
Uso de polímeros catiônicos e aniônicos como produtos auxiliares no tratamento de água
}

\author{
Use of cationic and anionic polymers as additional products \\ in water treatment plants
}

\author{
Eduardo Luiz Delmonico ${ }^{1}$, Arcioni Ferrari Constantino ${ }^{2}$, Charles Carneiro ${ }^{3}$ \\ Recebido: 01/09/2020. Aceito: 23/10/2020
}

\begin{abstract}
Resumo
Os procedimentos de coagulação, floculação e decantação têm, cada vez mais, assumido maior importância operacional em processos de tratamento de água, em razão da ótima relação custo-benefício. Quando a incidência de chuvas aumenta e, consequentemente, a turbidez da água in-natura dos rios destinados ao abastecimento das cidades, há necessidade do emprego de novas e mais eficientes tecnologias no tratamento, como por exemplo, o emprego de polímeros auxiliares de floculação. O presente trabalho teve como objetivo avaliar o uso de polímeros catiônico e aniônico como produtos auxiliares ao policloreto de alumínio (PAC) no processo de coagulação e floculação da Estação de Tratamento de Água (ETA) Pirapó, Maringá - PR. A turbidez da água foi analisada sob as seguintes condições: i) somente PAC; ii) PAC + polímero auxiliar aniônico; iii) PAC + polímero auxiliar catiônico. A quantidade de sólidos gerada e aspectos econômico-financeiros da utilização dos polímeros auxiliares também foram avaliados. A adição do polímero apresentou resultados bastante satisfatórios, alcançando 99,96\% de remoção de turbidez (0,26 NTU na água produzida); com melhor desempenho quanto maior a turbidez da água in-natura. Também houve uma redução da necessidade de limpeza de decantadores proporcionada pela maior redução de turbidez associada à presença do polímero auxiliar. Quanto ao lodo, naturalmente como esperado, observou-se maior produção em função da maior turbidez (períodos chuvosos). A redução de custos no processo de tratamento ocasionada pelo uso dos polímeros catiônico e aniônico variou entre 17 e $21 \%$.
\end{abstract}

Palavras-chave: Tratamento de água. Polímero. Floculação.

\begin{abstract}
The processes of coagulation, flocculation and decanting have increasingly assumed greater importance from the operational point of view because it has a better price-performance ratio in water treatment systems. When the incidence of rain increases and consequently the turbidity of in-nature water from rivers destined to supply cities, it is necessary to use new e efficient technologies, such as the use of auxiliary flocculation polymers. The present work aimed to assess the use of cationic and anionic polymers as auxiliary products of the coagulation and flocculation process. The turbidity performance was evaluated as follow: i) only PAC coagulant, ii) PAC + anionic auxiliary polymer; and iii) PAC + cationic auxiliary polymer. The amount of sludge generated due the polymers addition, as well as the costs of the employed products, were also assessed. The results showed excellent efficiency with the addition of the polymer. In conditions of high turbidity this importance is even greater, reaching $99,96 \%$ of turbidity reduction $(0,26$ NTU in the produced water). There also a significant decrease in the need for cleaning decanters provided by the greater reduction of turbidity with the auxiliary polymers. As for the sludge, naturally as expected, greater production was observed owing to the greater turbidity (rain periods). On the financial side, the addition of the cationic and anionic polymers generated saved costs between 17 and $21 \%$ compared to the traditional water treatment process.
\end{abstract}

Keywords: Water treatment. Polymer. Flocculation.

\footnotetext{
1 Mestre em Química pela Universidade Estadual de Maringá - UEM, Pós-Graduando em Saneamento Ambiental pela Universidade Estadual Norte Paraná - UENP, Agente de suporte operacional pela Sanepar. E-mail: profel.quimica@gmail.com

2 Químico licenciado pela Universidade Estadual de Maringá - UEM. Agente de suporte operacional pela Sanepar. E-mail: arcionifc@sanepar.com.br

3 Instituto Superior de Administração e Economia do Mercosul -ISAE e Secretaria de Estado do Desenvolvimento Sustentável e do Turismo do Paraná - SEDEST. E-mail: charles.c@sedest.pr.gov.br 


\section{Introdução}

Dentre as rotas tecnológicas mais utilizadas para tratamento de águas de consumo no Brasil a associação entre coagulação/floculação/decantação é uma das mais utilizadas, em função do ótimo custo-benefício.

Os principais efeitos causados pela adição de polímeros no tratamento de água são: o aumento do tamanho, da densidade e da resistência das partículas floculadas, melhorando assim a velocidade de decantação dos flocos e aumentando a resistência destes ao cisalhamento causado pelo escoamento (LIBÂNIO, 2008; RICHTER, 2009). As partículas eletricamente desestabilizadas (coágulos) são fisicamente aglutinadas para formar outras partículas maiores (flocos), suscetíveis de remoção por decantação e filtração (MACEDO, 2001). A floculação pode ocorrer pela própria movimentação hidráulica e/ou movimentação mecânica. Os polímeros atuam favorecendo a formação de pontes de hidrogênio e/ou forças de Van der Walls. A robustez e densidade do floco são influenciadas pelo "gradiente de velocidade", que varia entre plantas de tratamento (AMIRTHARAJAH \& O'MÉLIA, 1990). O material acumulado forma o lodo de água, que deve ser removido mecanicamente ou por meio de lavação do decantador (JORDÃO \& PESSOA, 1995). O lodo gerado pode representar um volume significativo em relação ao volume de água tratado (ACHON et. al., 2013) e deve receber uma gestão ambientalmente adequada; ademais, deve atender os preceitos da Política Nacional de Resíduos Sólidos - Lei 12.305/2010 (BRASIL, 2010).

Buscando melhoria de desempenho e técnicas mais sustentáveis em processos de coagulação/floculação/ decantação, rota tecnológica predominante nos sistemas de tratamento de água, produtos naturais têm sido testados. A fécula de mandioca foi utilizada como auxiliar de floculação para aumento da capacidade no tratamento de água na cidade de Valinhos - SP, e apresentou melhora do sistema de floculação e sedimentação (SOUZA, 2003). Já estudos de SILVA (2019), testando a fécula em conjunto com cloreto férrico, não foram observados resultados positivos. O polímero do quiabo foi o objetivo do estudo de ABEU LIMA (2007), resultando em melhora nos valores de água decantada e gerando custo inferior, se comparado a outros polímeros naturais, entretanto ainda sugerindo a necessidade de mais testes.

Os polímeros catiônico e aniônico têm sido utilizados em pós-tratamento de efluentes industriais de papel e celulose, ocasionando melhoras nas características do efluente final (QUARTAROLI et al., 2014). Nas unidades de tratamento de água Iraí e Passaúna, o polímero aniônico foi utilizado aumentando os índices de clarificação e de consistência do lodo. Ainda na planta Iraí, o polímero catiônico foi utilizado melhorar a remoção de microalgas na água bruta (SANEPAR, 2011). REALI (1999) observou aumento no teor de sólidos de lodo de água cerca de 10 vezes com o emprego do polímero catiônico, passando de $0,6 \%$ para $6 \%$ de sólidos sedimentáveis.

Por outro lado, ainda são poucos os estudos que comparam valores de turbidez de água decantada em função da adição de polímeros aniônico e catiônico. Assim, uma melhor compreensão sobre o processo, bem como, a recente necessidade de aumento nos volumes a serem captados pelos sistemas nos últimos anos, têm motivado novos estudos por rotas tecnológicas alternativas, como o uso de polímeros auxiliares de floculação. 
O frequente uso do coagulante PAC, em muitas situações, não tem se mostrado suficientemente efetivo na remoção de turbidez da água in-natura, e por vezes, elevando valores de turbidez da água decantada e filtrada, comprometendo o tratamento de água.

Considerando que cada unidade operacional requer sua própria configuração de trabalho - dosagem, concentração, frequência, subprodutos, custos, entre outros, o presente trabalho teve como objetivo avaliar o uso de polímeros catiônico e aniônico como produtos auxiliares ao já utilizado PAC, no processo de coagulação e floculação em uma planta de tratamento de água localizada em Maringá - PR.

\section{Materiais e métodos}

\subsection{Caracterização da área de estudo}

A Estação de Tratamento de Água Pirapó (ETA-Pirapó), utilizada como estudo de caso, está localizada nas coordenadas $23^{\circ} 24^{\prime} 344^{\prime \prime}$ e e 51 55'26.6”W, cidade de Maringá- PR, e capta água na bacia do Rio Pirapó, com vazões variando entre $400 \mathrm{~L} / \mathrm{s}$ e $1.440 \mathrm{~L} / \mathrm{s}$.

O rio Pirapó é enquadrado como classe 2 (SUREHMA, 1991), de acordo com a resolução CONAMA 357/2005(BRASIL, 2005). O rio é considerado perene, e teve sua vazão máxima de $32.000 \mathrm{~L} / \mathrm{s}$ registrada no ano de 2014. Atualmente, 93 milhões de litros são captados diariamente para suprimento humano (CÂMARA MUNICIPAL DE MARINGÁ, 2015). A Tabela 1 apresenta a precipitação média e turbidez média do rio Pirapó durante o ano de 2019.

Tabela 1 - Precipitação pluviométrica e turbidez média registrada na água

in natura - Bacia rio Pirapó - 2019

\begin{tabular}{|c|c|c|c|c|c|c|c|c|c|c|c|}
\hline & JAN & FEV & MAR & ABR & MAI & JUN & JUL & AGO & SET & OUT & NOV \\
\hline $\begin{array}{l}\text { Precipitação mé- } \\
\text { dia }(\mathrm{mm})\end{array}$ & 100,6 & 109,6 & 123,8 & 29,0 & 60,4 & 2,8 & 33,8 & 1,0 & 49,4 & 62,6 & 31,6 \\
\hline $\begin{array}{l}\text { Turbidez } \\
\text { média (NTU) }\end{array}$ & 152 & 161 & 241 & 28 & 55 & 12 & 17 & 13 & 24 & 59 & 74 \\
\hline
\end{tabular}

Fonte: Os autores, 2020.

A água in natura do rio Pirapó, durante o período de estudo, apresentou as seguintes variações físico-químicas: pH 7,20 a 7,80; turbidez 23 a 993 NTU; cor 100 a $5000 \mathrm{uH}$; e alcalinidade 42 a 54 mg L-1 $\mathrm{CaCO}_{3}$.

\subsection{Caracterização dos ensaios analíticos}

O estudo foi desenvolvido entre abril e novembro de 2019, e buscou avaliar a melhor opção de polímero auxiliar - aniônico ou catiônico - que deve ser aplicado junto ao coagulante policloreto de alumínio (PAC) no tratamento convencional da ETA-Pirapó. O estudo comparou valores de decantação obtidos no processo de floculação para os seguintes tratamentos: i) apenas o coagulante PAC, ii) PAC + polímero catiônico e, iii) 
PAC + polímero aniônico, e sob diferentes tempos de aplicação: 0 minuto; 5 minutos; 7 minutos; e 9 minutos.

O principal parâmetro indicador de performance analisado foi turbidez: quanto mais baixo o valor de turbidez observado, melhor o desempenho alcançado. Para auxiliar na avaliação de desempenho dos tratamentos, os parâmetros $\mathrm{pH}$, cor e alcalinidade também foram determinados. Uma sequência de 11 amostragens (enumeradas de 1 a 11) de água in natura foi realizada antes e durante um episódio de chuva, buscando obter a maior variabilidade possível de turbidez na água bruta.

Para os ensaios de floculação, utilizou-se equipamento de Jar-Test JT303M - Milan ${ }^{\circledR}$. As configurações estabelecidas nos equipamentos de jar-test para as análises foram semelhantes às regularmente utilizadas no tratamento de água na unidade operacional Pirapó e podem ser encontram na Tabela 2.

A concentração do PAC no ensaio de floculação foi $2 \%(\mathrm{~m} / \mathrm{V})$, enquanto que a concentração imposta nos polímeros catiônico e aniônico foi $0,02 \%(\mathrm{~m} / \mathrm{V})$.

Tabela 2 - Configuração do Jar-test utilizada nos ensaios de floculação

\begin{tabular}{ll}
\hline AGITAÇÃO & TEMPO \\
\hline Rápida $-100 \mathrm{rpm} *$ & 15 segundos \\
Lenta $-60 \mathrm{rpm}$ & 10 minutos \\
Decantação & 10 minutos \\
\hline
\end{tabular}

NOTA: *rpm: rotações por minuto.

Fonte: Os autores, 2020.

\subsection{Quantificação do lodo}

Para quantificar o lodo gerado no processo foram realizadas análises do teor de sólidos sedimentáveis (SS) em cada uma das 11 amostragens de água in natura.

As análises foram performadas com o auxílio de cones Imhoff, e após os testes em jar-test, como segue: repouso por 45 minutos, leve agitação com ajuda de bastão de vidro, posteriormente decantação 15 minutos, e então realização das respectivas marchas analíticas.

\subsection{Avaliações econômico-financeiras}

Para verificar a viabilidade financeira do emprego dos polímeros auxiliares no processo de tratamento convencional da água foi realizada uma avaliação simples de custos.

A partir da estimativa do custo da implementação das estruturas de aplicação dos polímeros (R\$), somadas a quantidade $(\mathrm{kg})$ de cada insumo participante do processo, polímeros auxiliares cationnico e aniônico e PAC, e comparou-se com o procedimento tradicional de tratamento que faz uso somente de PAC. 


\section{Resultados e discussão}

\subsection{Coagulação e floculação}

\subsubsection{Tempo de aplicação dos polímeros}

A avaliação teve início pela definição do tempo ideal para a adição do polímero auxiliar + PAC no processo de floculação. Foram feitos testes adicionando os polímeros catiônico e aniônico, juntamente com o PAC, nos seguintes tempos: 0 minuto; 5 minutos; 7 minutos; 9 minutos.

A maior eficiência no processo de decantação foi observada quando os polímeros foram adicionados no tempo "9 minutos" de floculação: turbidez 1,66 NTU - Catiônico e 1,59 NTU - Aniônico. Quanto menor o valor de turbidez obtido na água decantada, melhor o resultado do tratamento de água. Assim, esse tempo foi definido para a continuidade dos testes de concentração.

\subsubsection{Otimização da dosagem dos polímeros}

As concentrações dos polímeros catiônico e aniônico testadas foram: 0,04;0,06;0,08;0,10;0,30;0,50; 0,70; e 0,90 mg L-1. O Gráfico 1 apresenta os valores de decantação (turbidez NTU) obtidos em função da dosagem utilizada dos polímeros.

Gráfico 1 - Valores de turbidez no processo de decantação em função de diferentes concentrações dos polímeros catiônico e aniônico

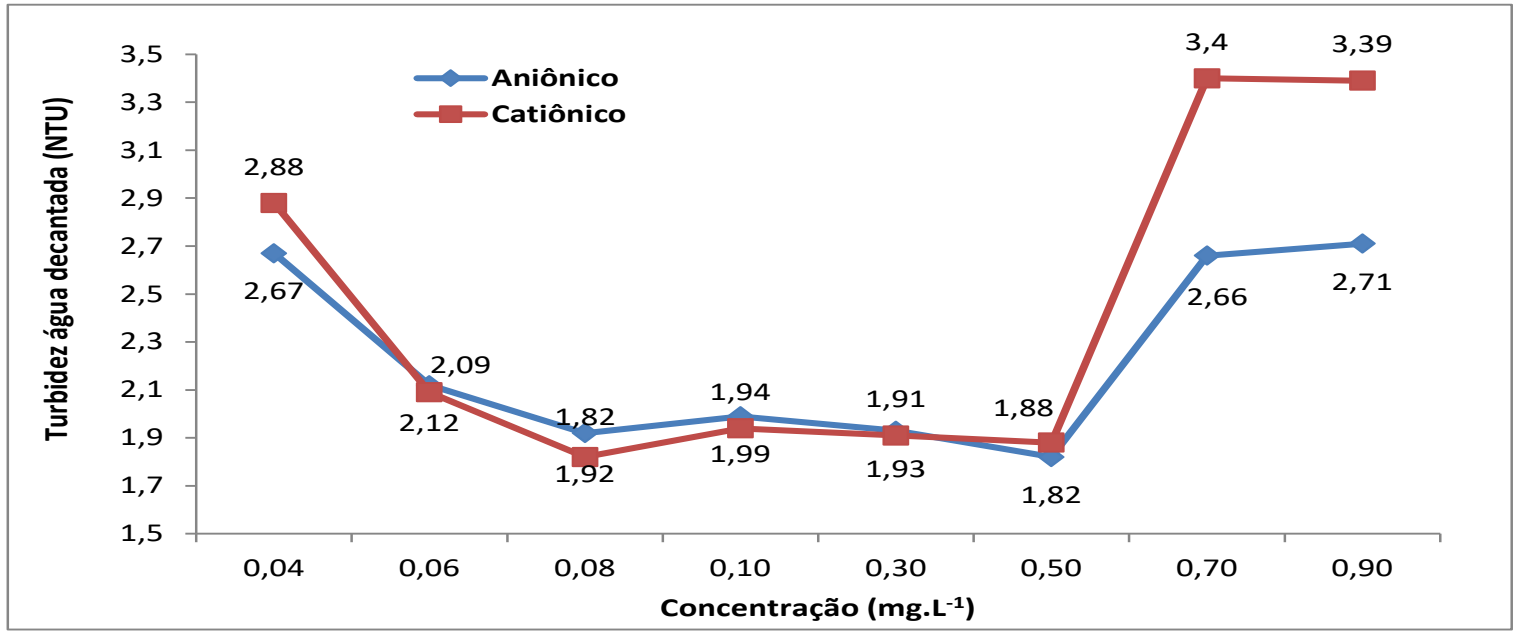

Fonte: Os autores, 2020.

Entre o intervalo de doses testadas de 0,08 a $0,50 \mathrm{mg} \mathrm{L}^{-1}$ obteve-se os melhores resultados em termos de turbidez da água decantada (1,92 a 1,99 NTU), e com valores muito próximos nesta amplitude de dosagens, sugerindo aí um intervalo ótimo de concentração de ambos os polímeros.

Com isso, selecionou-se a concentração de polímero de $0,10 \mathrm{mg} \mathrm{L}^{-1}$, próxima do valor médio do in- 
tervalo supracitado, para a continuidade dos testes. Sob o aspecto financeiro, esta dosagem potencialmente significaria uma utilização menor de insumos no tratamento da água.

\subsubsection{Desempenho dos polímeros}

Foram avaliadas diferentes concentrações de água bruta, desde valores mais baixos característicos de estiagem $(<50 \mathrm{NTU})$, até valores mais elevados, acima de 900 NTU, característicos de períodos chuvosos. A tabela 2 apresenta os valores de turbidez gerados após a aplicação dos tratamentos com os polímeros - concentração de $0,10 \mathrm{mg} \mathrm{L}^{-1}$, comparativamente à turbidez da água in natura.

Conforme apresenta a Tabela 3, o polímero aniônico proporcionou o melhor desempenho nos testes realizados, alcançando menores valores de turbidez comparativamente aos demais tratamentos. A extrapolação disto para uma escala operacional significaria ganhos significativos no processo, já que os filtros teriam sua capacidade filtrante aumentada com a diminuição da turbidez da água decantada, além de prováveis ganhos financeiros.

Tabela 3 - Valores de turbidez (NTU) da água decantada com polímero catiônico + PAC, polímero aniônico + PAC e somente PAC, em função de diferentes valores de turbidez na água in natura

\begin{tabular}{llllllllllll}
\hline Amostra & $\mathbf{1}$ & $\mathbf{2}$ & $\mathbf{3}$ & $\mathbf{4}$ & $\mathbf{5}$ & $\mathbf{6}$ & $\mathbf{7}$ & $\mathbf{8}$ & $\mathbf{9}$ & $\mathbf{1 0}$ & $\mathbf{1 1}$ \\
\hline $\begin{array}{l}\text { Água } \text { in-natura } \\
\text { Água decantada c/ }\end{array}$ & 25 & 39 & 125 & 194 & 244 & 312 & 450 & 578 & 774 & 890 & 993 \\
$\begin{array}{l}\text { PAC + Polímero } \\
\text { Aniônico }\end{array}$ & 1,9 & 2,7 & 2,1 & 1,3 & 1,5 & 1,7 & 3,3 & 2,1 & 2,1 & 3,3 & 3,7 \\
$\begin{array}{l}\text { Água decantada c/ } \\
\text { PAC + Polímero }\end{array}$ & 1,9 & 3,4 & 4,2 & 2,4 & 2,8 & 2,9 & 4,8 & 3,6 & 3,9 & 3,4 & 4,7 \\
$\begin{array}{l}\text { Catiônico } \\
\text { Água decantada c/ }\end{array}$ & 2,5 & 4,0 & 2,4 & 2,2 & 2,5 & 3,8 & 5,4 & 5,5 & 6,0 & 6,0 & 6,8 \\
PAC & & & & & & & & & & & \\
\hline
\end{tabular}

Fonte: Os autores, 2020.

Este melhor desempenho do polímero aniônico, assim como do catiônico, também pode ser percebida por meio de análise visual da água com os flocos formados, conforme ilustra, por exemplo, o ensaio de jar-test com a água in natura de 312 NTU (Figura 1). Percebe-se que a água floculada que recebeu os polímeros auxiliares tem aspecto mais robusto em termos de tamanho e volume do floco formado, isto possibilita um processo de decantação mais acelerado. Além disso, nota-se uma formação de lodo mais coeso quando os polímeros auxiliares são adicionados (ver figura 1). 
Figura 1 - Imagens dos tratamentos exemplificadas pelo jar-test de água in natura- 312 NTU, sendo: A - processo de floculação; B - início da decantação; C - lodo após a decantação

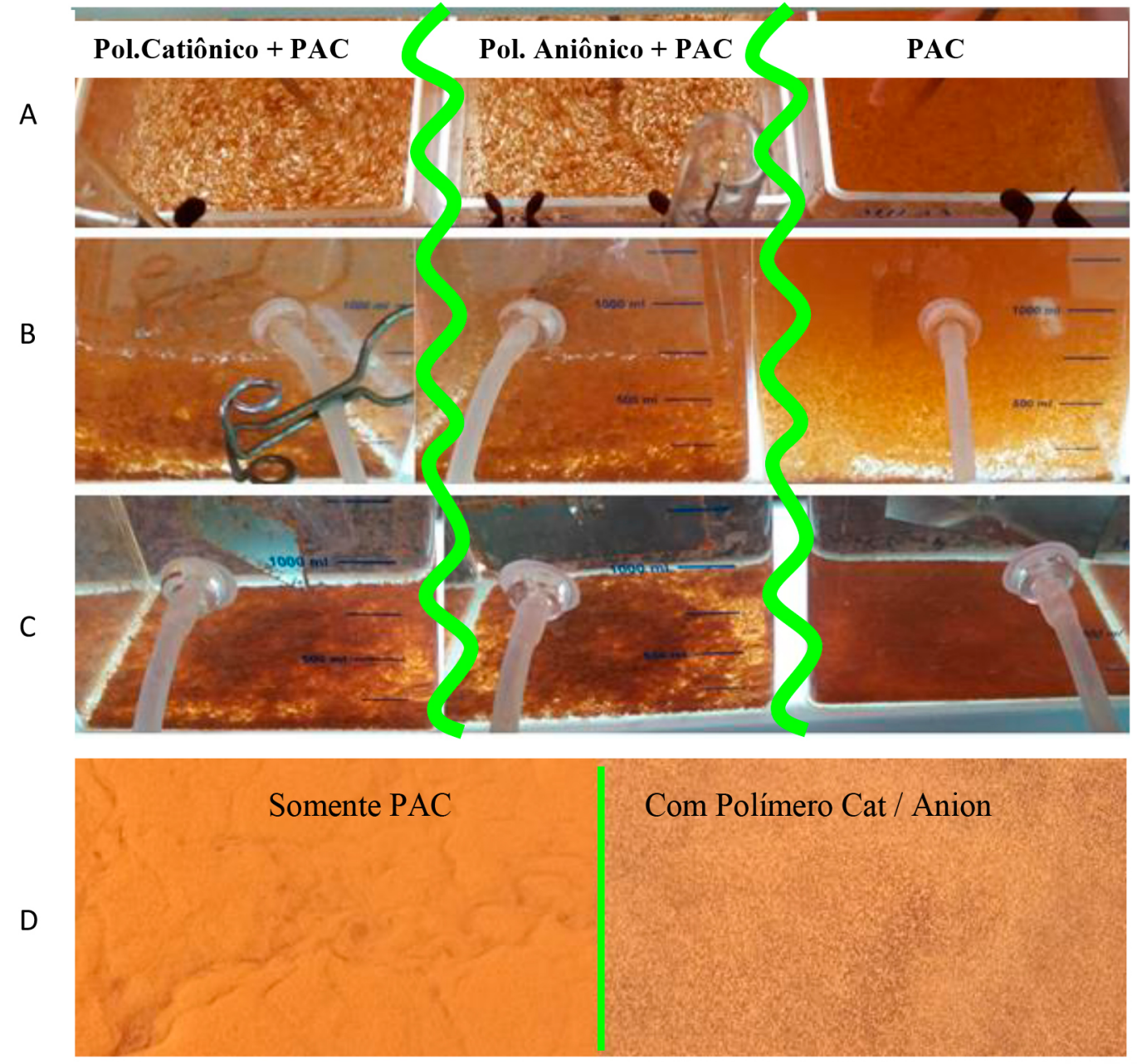

Fonte: Os autores, 2020.

Um breve período de operação em escala (novembro 2019) na Estação de Tratamento de Água Pirapó, utilizando o polímero auxiliar catiônico junto ao PAC, promoveu uma redução de turbidez ainda maior do que a já eficiente desempenho de PAC somente. Com o emprego de PAC a redução média de turbidez na água decantada foi de 99,24\%, e que pós-filtragem física a redução final foi de 99,91\%, ou seja, passando de 681 NTU na água in natura, para 0,61 NTU na água produzida final, um bom desempenho, mas ainda não atendendo a legislação. A Portaria Consolidada 05/2017 (Ministério da Saúde, 2017) preconiza valores <0,5NTU como limite de turbidez para água de consumo.

Já o desempenho de PAC + polímero auxiliar foi superior, a redução média na água de decantação foi de $99,74 \%$ e pós-filtragem física a redução final foi de 99,96\%, isto é, passando de 653 NTU na água in natura, para 0,26 NTU na água produzida final e conseguindo atender plenamente os padrões de potabilidade legalmente preconizados. 


\subsection{Quantificação do lodo}

A ETA-Pirapó dispunha de 12 decantadores que comportavam o lodo gerado processo de tratamento, a partir de uma vazão média de adução de $80.000 \mathrm{~m}^{3}$ dia $^{-1}$. O Gráfico 2 permite comparar os quantitativos de lodo produzidos nos testes de bancada (Imhoff), em relação à quantidade de lodo gerado $\left(\mathrm{kg}\right.$ dia $\left.^{-1}\right)$ durante um dia de trabalho em escala operacional na ETA-Pirapó, a partir da mesma água in-natura captada.

Gráfico 2 - Valores de geração de lodo (teor de SS) obtidos nos testes com cones imhoff, e durante um dia de trabalho em escala operacional $\left(\mathrm{kg} \mathrm{dia}^{-1}\right)$, em função de diferentes valores de turbidez da água in natura

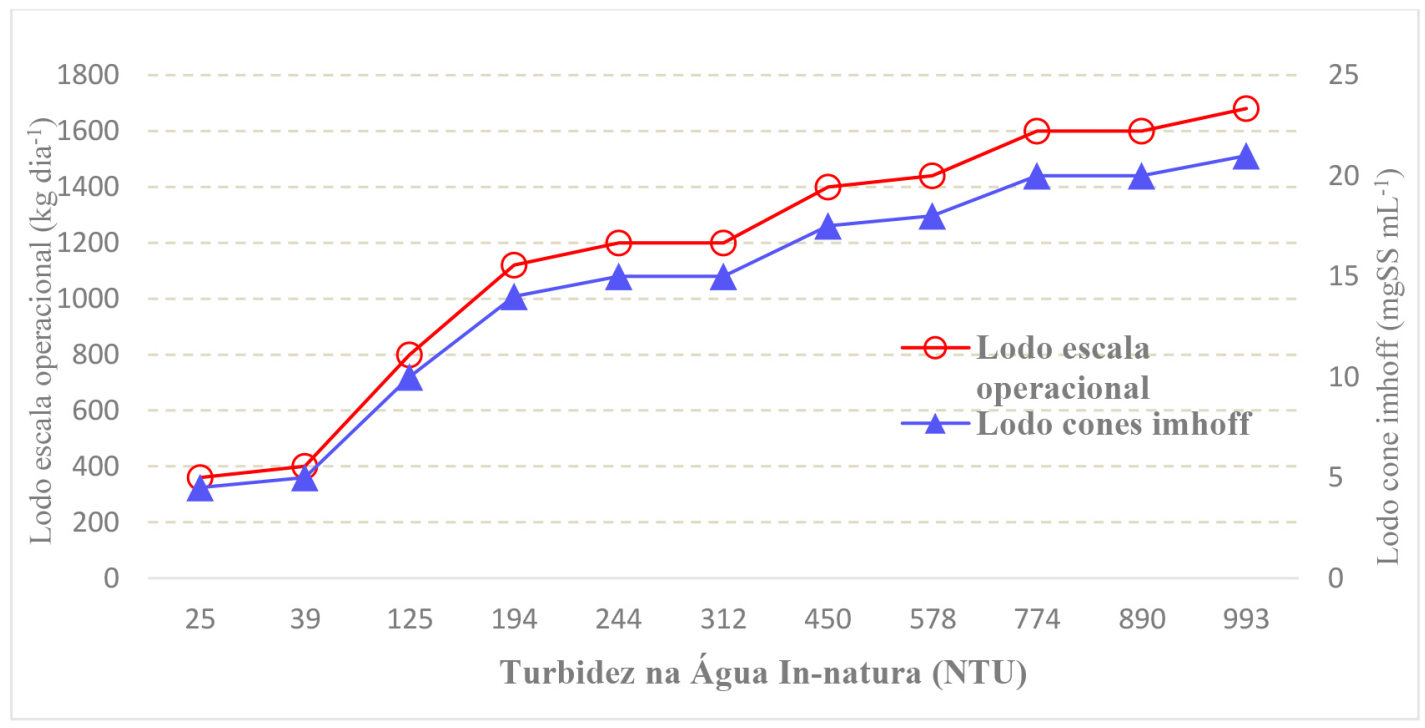

Fonte: Os autores, 2020.

Com o aumento da turbidez da água in natura a quantidade de lodo gerado também aumentou de maneira proporcional, próxima da linearidade. O estudo constatou que o lodo gerado a partir de uma turbidez in natura da ordem de 25 NTU permitiria uma capacidade de trabalho da ordem de 5 dias; por outro lado, se a turbidez estivesse bastante elevada, como por exemplo, próximo a $1000 \mathrm{NTU}$, a capacidade de trabalho do decantador seria inferior a 1 dia, necessitando limpeza. Logo, há possibilidade de aperfeiçoar o sistema de lavagem de decantadores e também constituir um sistema de descarga de lodo a fim de aumentar a eficiência do sistema e diminuir perdas no sistema produtor.

Verificou-se também que, independentemente do uso ou não dos polímeros auxiliares, os valores de concentração de constituintes do lodo não sofreram alterações.

\subsection{Avaliação financeira do uso dos polímeros}

Avaliou-se a redução da quantidade de coagulante utilizada no tratamento de água mantendo-se constante a concentração dos polímeros. O uso dos polímeros, em tese, resultaria em um custo inferior ao praticado 
apenas com o coagulante PAC.

Para a avaliação de custos da adição de polímeros, considerou-se:

- Custo unitário do PAC $=1,64 \mathrm{R} \$ / \mathrm{kg}$

- Custo unitário do polímero aniônico $=8,38 \mathrm{R} \$ / \mathrm{kg}$

- Custo unitário do polímero catiônico $=18,12 \mathrm{R} \$ / \mathrm{kg}$

- Média de água produzida por dia $=65.530 \mathrm{~m}^{3}$

- Média de turbidez da água in natura= 209 NTU

- Valores de mercado referentes a novembro 2019

Logo, o custo da adição de polímeros, considerando 01 dia de tratamento, foi:

- Valor gasto no tratamento utilizando apenas PAC $(20 \mathrm{ppm})=\mathrm{R} \$ 7.157,45$

- Valor gasto no tratamento utilizando PAC $(15 \mathrm{ppm})+$ polímero aniônico $=\mathrm{R} \$ 5.644,63$

- Valor gasto no tratamento utilizando PAC $(15 \mathrm{ppm})+$ polímero catiônico $=\mathrm{R} \$ 5.966,05$

Em ambos os casos, o uso de polímeros, catiônico ou aniônico, representou uma economia significativa em relação ao uso apenas do coagulante PAC. A combinação "polímero catiônico + PAC" resultou em uma redução de R \$ 1.191,40 por dia, comparativamente ao uso isolado de PAC. Já a combinação "Polímero Aniônico + PAC" gerou uma economia ainda maior, R \$1.512,82 por dia, além do já supramencionado, ganho significativo de eficiência em termos de redução de turbidez da água decantada.

\section{Considerações finais}

A utilização dos polímeros catiônico e aniônico como auxiliares para melhorar o desempenho do processo de floculação proporcionou uma maior eficiência no processo de tratamento da água, sendo superior ao tradicional uso apenas do policloreto de alumínio - PAC, alcançando com os polímeros catiônico e aniônico acima de $99,9 \%$ de remoção de turbidez, e colocando a água produzida final (0,26 NTU) com segurança dentro dos padrões de potabilidade (0,50 NTU - Portaria Consolidada n 05/2017 do Ministério da Saúde).

Os resultados obtidos para a quantificação do lodo mostraram que quanto maior os valores de turbidez da água in natura, maior a quantidade de lodo gerada. Os quantitativos de lodo de água gerados variaram de $360 \mathrm{~kg} \mathrm{dia}^{-1}$ em período de baixa pluviometria, chegando até $2.000 \mathrm{~kg} \mathrm{dia}^{-1} \mathrm{em}$ períodos chuvosos. Esta diferença influencia a necessidade de limpeza dos decantadores, uma vez que é possível trabalhar cerca de 5 dias com turbidez de $25 \mathrm{NTU}$, versus 01 dia com turbidez próxima a $1.000 \mathrm{NTU}$.

O uso dos polímeros catiônico e aniônico de forma auxiliar se mostrou viável economicamente, uma vez que foi possível reduzir cerca de $25 \%$ da quantidade de coagulante PAC convencionalmente utilizada no sistema de tratamento, o que resultou em diminuição de gastos entre 17 e $21 \%$ (em reais). 


\section{Referências}

ABREU LIMA, G.J. Uso de Polímero Natural do Quiabo como Auxiliar de Floculação e Filtração em Tratamento de Água e Esgoto. Dissertação (Mestre em Saneamento Ambiental) - Programa de Pós-graduação em Engenharia Ambiental, Universidade do Estado do Rio de Janeiro, Rio de Janeiro, 2007.

ACHON, C.L; BARROSO, M.M.; CORDEIRO, J.S. Resíduos de estações de tratamento de água e a ISO 24512: desafio do saneamento brasileiro. Engenharia Sanitária e Ambiental, 18, 2, p. 115-122, 2018.

AMIRTHARAJAH, A.; O'MÉLIA, C.R. Coagulation processes: destabilization, mixing and flocculation. Water quality and treatment. Toronto. Ed. McGrawHill: Canadá, 1990.

BRASIL. Ministério do Meio Ambiente. Conselho Nacional do Meio Ambiente CONAMA - Resolução 357 de 17 de março de 2005. Brasília, DF, 2005.

BRASIL. Lei No 12.305 de 02 de agosto de 2010. Política Nacional de Resíduos Sólidos. Congresso Nacional, Brasília, DF, 2010.

BRASIL. Ministério da Saúde. Portaria da Consolidação N5 de 28 de setembro de 2017. Brasília, DF. Disponível em: https://portalarquivos2.saude.gov.br/images/pdf/2018/marco/29/PRC-5-Portaria-de-Consolida----o-n---5--de-28-de-setembro-de-2017.pdf, 2017. Acesso em: 01 set. 2019.

CÂMARA MUNICIPAL DE MARINGÁ. Comissão Especial de Estudos Sobre a Água. Maringá, PR, 2015.

CARNEIRO, C.; ANDREOLI, C.V. Lodo de Estações de Tratamento de Água: gestão e Perspectivas Tecnológicas. $1^{\circ}$ Edição. Curitiba: SANEPAR, 2013.

COMPANHIA DE SANEAMENTO DO PARANÁ - SANEPAR. Sistema Interno da Sanepar - SCG. 2011.

JORDÃO, E.P.; PESSOA, C.A. Tratamento de esgotos domésticos. 3 ed. Rio de Janeiro, Associação Brasileira de Engenharia Sanitária e Ambiental-ABES, 720 p., 1999.

LIBÂNIO, M. Fundamentos de qualidade e tratamento de água. 2. Ed. Campinas: São Paulo, 2008.

MACEDO, J.A.B. Águas \& águas. São Paulo: Livraria Varela, 2001.

QUARTAROLI, L.; KURITZA, J.C.; CAVALLINI, G.S.; VIDAL, C.M.S.; SOUZA, J.B. Aplicação de polímeros aniônico e catiônico na flotação por ar dissolvido como forma de pós-tratamento de efluente de indústria de papel e celulose. Scientia Forestalis, 42, 101, p. 57-67, mar. 2014.

SILVA, L.E.F. Análise da aplicação do polímero de fécula de mandioca como floculante auxiliar do cloreto férrico no tratamento de água por sedimentação. Monografia (Graduação em Engenharia Química) - Universidade Federal do Rio Grande do Norte, Natal, 2019. 
SOUZA, L.C.A. Aumento da capacidade da estação de tratamento de água $\mathrm{N}^{\circ} \mathbf{2}$ de Valinhos-SP, utilizando polímero natural como auxiliar de floculação. Dissertação (Mestre em Hidráulica e Saneamento) - Escola de Engenharia de São Paulo, Universidade de São Paulo, São Paulo, 2003.

SUREHMA. Portaria nº 004, de 21 de março de 1991.

REALI, M.A.P. Principais Características Quantitativas e Qualitativas do Lodo de ETA's. In: CARNEIRO, C. \& ANDREOLI C.V. Lodo de Estações de Tratamento de Água: Gestão e Perspectivas Tecnológicas. Curitiba: SANEPAR, 2013.

RICHTER, C.A. Água: métodos e tecnologia de tratamento. Ed. Edgard Blücher: São Paulo, 2008. 